\title{
Eosinophilic peritonitis in a patient with liver cirrhosis: A case report
}

\author{
Mengyuan Pang ${ }^{1}$, Fang Hua ${ }^{2}$, Yixiao $\mathrm{Zhi}^{1}$, Eryun Qin ${ }^{1}$, Yu Tao ${ }^{1}$, Rui Hua*1 \\ ${ }^{1}$ Department of Hepatology, the First Hospital of Jilin University, China \\ ${ }^{2}$ Department of Cardiovascular, the First Hospital of Jilin University, China
}

Received: February 28, 2019

Accepted: May 26, 2019

Online Published: June 4, 2019

DOI: $10.5430 /$ crcp.v6n1p5

URL: https://doi.org/10.5430/crcp.v6n1p5

\begin{abstract}
Eosinophilic gastroenteritis (EG) is a gastrointestinal disease characterized by abnormal infiltration of eosinophilic cells in the gastrointestinal tract, excluding known causes of eosinophilia. Eosinophilic peritonitis (EP) is rare and is considered by most scholars to be a systemic or local allergy to exogenous or endogenous allergens. It is a clinical manifestation of eosinophilic gastroenteritis involving the serosal layer. Hereby, we report a case of EP in a patient with liver cirrhosis. A 32-year-old man was admitted to our hospital for intermittent fatigue, abdominal distension and abdominal pain. On account of clinical feature and pathological results of peritoneal puncture biopsy, excluding other causes of peripheral eosinophilia, the diagnosis of EP with hepatic cirrhosis was established. The possibility of EP should be paid great attention to patients with cirrhosis with peritonitis. Gastrointestinal endoscope biopsy, laparoscopy or peritoneal puncture biopsy are conducive to the diagnosis and differential diagnosis. Therefore, once the disease is suspected, gastrointestinal endoscope biopsy should be performed actively, and multiple pathological samples should be taken to contribute to diagnosis and treatment. Laparoscopy or peritoneal puncture biopsy is of vital significance for definitive diagnosis.
\end{abstract}

Key Words: Eosinophilic peritonitis (EP), Eosinophilic gastroenteritis (EG), Liver cirrhosis, Corticosteroid therapy

\section{INTRODUCTION}

Eosinophilic gastroenteritis (EG) refers to eosinophilic gastrointestinal disorders, which includes eosinophilic esophagitis, gastritis, enteritis and colitis. ${ }^{[1]}$ Eosinophilic peritonitis (EP) is a clinical feature of eosinophilic gastroenteritis involving the serosal layer, which is a rare, benign inflammatory disorder of the gastrointestinal tract. The estimated prevalence reported for eosinophilic gastroenteritis is $1 / 100,000$, however given the rarity of the diagnosis it is yet underestimated. ${ }^{[2]}$

According to different layers of eosinophils infiltration in the gastrointestinal wall, EG is classified into mucosal, muscular and serous lesions by Klein and so on. ${ }^{[3]}$ Its clinical features are as follows: (1) mucosal lesions: It is the most common type in clinical practice, characterized by abundant eosinophils infiltration in mucosa and manifesting as indigestion mainly. (2) muscular lesions: It is relatively rare in clinical practice, characterized by abundant eosinophils infiltration in muscularis. And the gastrointestinal wall is thickened, stiff and nodular, which can lead to intestinal obstruction. (3) serosal layer lesions: It is rare in clinical practice, characterized by abundant eosinophils infiltration in serous membrane. And it can lead to peritonitis, ascites and a large number of eosinophils in ascites smear. Subserosal infiltration of eosinophils can be seen by laparoscopy or laparotomy biopsy.

*Correspondence: Rui Hua; Email: hr1116@ sina.com; Address: Department of Hepatology, the First Hospital of Jilin University, China. 


\section{Presentation of Case}

\subsection{Clinical presentation}

A 32-year-old Chinese man was recommended to our hospital in December 2012 because of intermittent fatigue for 13 years, abdominal distension and abdominal pain for 14 days. History of present illness: the patient suffered from intermittent fatigue and was found that hepatitis B etiology positive for more than 13 years. He was admitted to our hospital for the aggravation of fatigue 10 months ago before this admission. Liver CT showed a cirrhotic liver and no ascites. Lamivudine combined with Adefovir was administered as an anti-viral therapy after the diagnosis of hepatitis B cirrhosis being established. He was admitted to local hospital for fever after dinner (up to $38.5^{\circ} \mathrm{C}$ ), anorexia, abdominal distension and abdominal pain and liver protection and anti-infection treatment were performed, but the above symptoms were not significantly relieved. He was recommended to our hospital for further diagnosis and treatment. There was no history of eating raw fish or raw meat, parasitic infection and allergy.

Physical examination: The patient was alert and showed no distress and was hemodynamically stable. Physical findings of skin and mucosa, the heart and lungs were normal. The abdomen was distended and tender diffusely with shifting dullness present. And rebound and guarding was observed. There was no splenomegaly, hepatomegaly and bilateral leg edema.

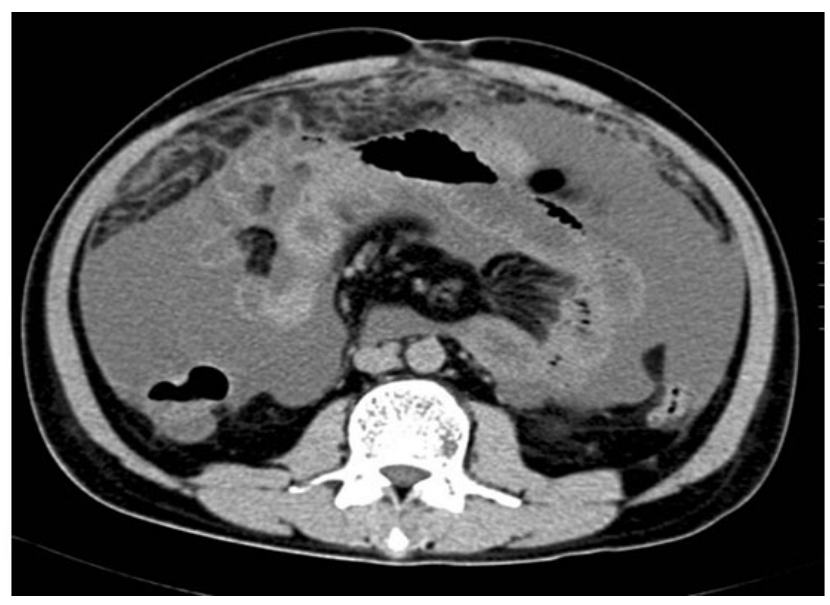

Figure 1. CT of the whole abdomen before hormone treatment: 1) diffuse changes of the omentum and thickening of the mesentery; 2) ascites

Laboratory tests: the count of eosinophil was $1.12 \times 10^{9} / \mathrm{L}$. The level of CRP $(110 \mathrm{mg} / \mathrm{L})$, ESR $(22 \mathrm{~mm} / \mathrm{h})$ and $\operatorname{IgE}$ $(558 \mathrm{IU} / \mathrm{ml})$ was elevated. Fecal egg test was negative. Analysis of the ascities fluid showed that the count of white blood cell ranged from $379 \times 10^{6} / \mathrm{L}$ to $2,350 \times 10^{6} / \mathrm{L}(75 \%$ mono- cytes, 25\% multicellular cells). The level of ADA and LDH was 5.20 U/L and $144 \mathrm{U} / \mathrm{L}$. Bacteria and fungus culture of ascites were negative. Noeosinophilic granulocytes were found in the classification of cells by ascites centrifugal sedimentation smear. TB antibody, PPD and TB test of ascites were negative. There was no cancer cell in ascites.

Imaging examination: Full abdominal CT plain scan and phase III enhanced scan (see Figure 1): (1) diffuse changes of the omentum and thickening of the mesentery; (2) sigmoid colon thickening slightly; (3) changes of ascites after abdominal drainage. Abdominal CT plain scan (see Figure 2) after hormone therapy: diffuse changes of omentum majus, intraperitoneal effusion and the amount of liquid decreasing slightly compared with the previous CT.

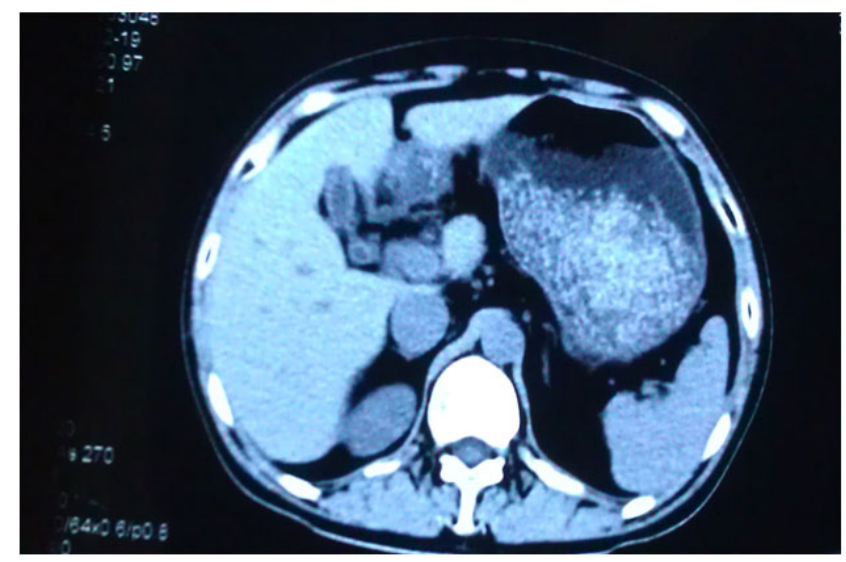

Figure 2. Abdominal CT (1 week after hormone therapy): Diffuse changes of omentum majus, intraperitoneal and retinal cystic effusion, the amount of liquid slightly decreased compared with the previous CT

\subsection{Pathological findings}

Gastroscopy and colonoscopy suggested chronic gastritis, non-atrophic and chronic colitis. Gastrointestinal biopsy revealed that chronic inflammation, edema of mucosa and bland hyperplasia could be seen in the area of duodenal descending, gastric antrum and gastric body. Bone marrow smear displayed that the count of eosinophils increased (7\% eosinophilic myelocyte, $4.5 \%$ eosinophilic metamyelocyte, $3.5 \%$ eosinophilic stab granulocyte, $7 \%$ eosinophil segmented cell). Bone marrow biopsy showed that the proliferation of nuclear cells decreased in bone marrow. Ultrasoundguided peritoneal puncture biopsies (see Figure 3) revealed that a large number of eosinophils were observed.

The patient, a middle-aged man with a long medical history, denied a history of parasitic infections and a history of food allergies. Clinical manifestations were inappetence, abdominal distension and abdominal pain. Abdominal palpation was 
tenderness and rebound pain. There was no infiltration of the eosinophils in gastrointestinal biopsy, but the infiltration of abundant eosinophils was eventually found in peritoneal puncture biopsy. According to the patient's medical history, symptoms, signs and pathological results, the diagnosis of EP could be established.

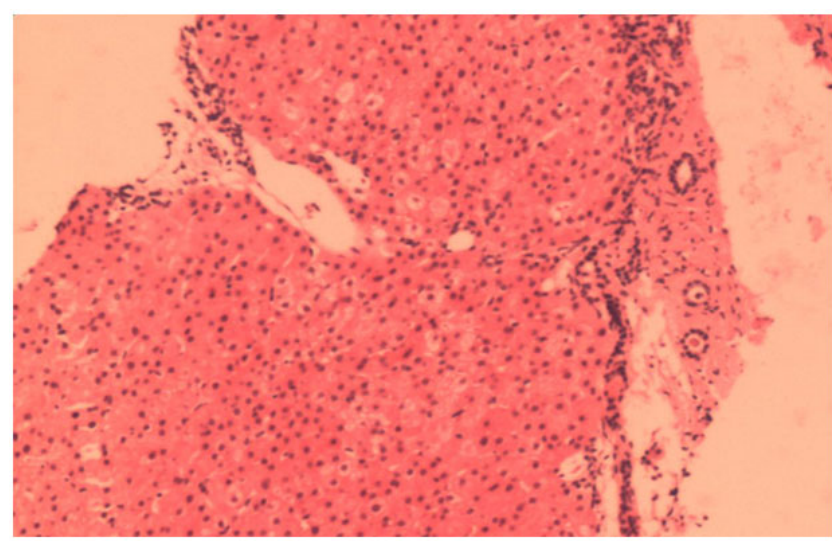

Figure 3. Omental biopsy via peritoneal puncture: Large Numbers of eosinophils were found in the omentum

Definite clinical diagnosis: hepatitis B cirrhosis, eosinophilic peritonitis. $30 \mathrm{mg} / \mathrm{d}$ of methylprednisolone was given on the basis of antiviral therapy after diagnosis being established. The clinical symptoms of the patient disappeared and the count of eosinophils in peripheral blood returned to normal after 1 week of treatment. The hormones gradually decreased after discharge, and the ascites completely subsided after 2 months.

\section{DisCUSSION}

Primary eosinophilic gastrointestinal is a benign inflammatory disorder that selectively affects the gastrointestinal tract with eosinophil-rich inflammation in the absence of known causes for eosinophilia (e.g., drug reactions, parasitic infections, and malignancy), which includes eosinophilic esophagitis, gastritis, enteritis and colitis. ${ }^{[1]}$ The diagnostic requirements are as follows: (1) gastrointestinal symptoms, (2) eosinophilic infiltration in one or more segments of the gastrointestinal tract, and (3) exclusion of other causes of intestinal eosinophilia. ${ }^{[4-6]}$

Eosinophilic peritonitis is a clinical manifestation of eosinophilic gastroenteritis involving the serosal layer which presents with isolated ascites or ascites with symptoms characteristic of mucosal or muscular EG. ${ }^{[7]}$ The diagnostic feature is a marked eosinophilia, up to 88 percent, in the ascetic fluid. ${ }^{[3]} \mathrm{EP}$ is a rare, specific type of noninfectious peritoneal dialysis-associated peritonitis which is often associated with Published by Sciedu Press either a reaction to the dialysis system components (catheters, disinfectants, or solution) or a potential bacterial or fungal reaction. Unlike other persistent infectious peritonitis, it is critical that dialysis catheter removal is not indicated. Shortterm oral administration of low-dose prednisone acetate has been recommended for patients to maintain catheter patency if the peritoneal fluid is markedly turbid. ${ }^{[8]}$

EP should be suspected in any patient with gastrointestinal symptoms associated with peripheral eosinophilia. It is necessary to rule out other diseases such as intestinal tuberculosis, drug reactions, parasitosis, and malignant neoplasms when the diagnosis of EP is established. In addition, gastrointestinal endoscope biopsy should be performed actively. However, according to the guideline published in 2014 by the Japanese Ministry of Health, Labor and Welfare, as negative endoscopic mucosal biopsy can't definitively rule out muscular or subserosal EG, laparoscopic full-thickness biopsy is required to confirm the diagnosis. ${ }^{[9]}$ We should consider the possibility of EP and perform laparoscopy or peritoneal puncture biopsy when nothing abnormal is found in gastrointestinal endoscopy biopsy. An early diagnosis and rapid application of corticosteroids may reduce the duration and severity of symptoms and thus reduce the morbidity of this disease.

The treatment is empiric and based on the severity of the clinical presentation. Patients who are symptomatic or have evidence of malabsorption may be treated with systemic glucocorticoids. ${ }^{[10]}$ The treatment with steroids shows an improvement in up to $90 \%$ of cases; however, the duration is not specified, and relapse is not uncommon; hence, treatment tends to prolonged. There is no consensus about the optimal type or dose of steroid; however, budesonide has the advantage of a local effect and a first step metabolism which entails less risk for adrenal suppression; the oral prednisolone dose most frequently administered is $40 \mathrm{mg} /$ day for 7-14 days, followed by a progressive dose reduction. In some cases, maintenance treatment with low doses ( 5 to $10 \mathrm{mg}$ a day) is necessary. ${ }^{[11]} \mathrm{H} 1$ receptor antagonist (diphenhydramine, ${ }^{[12]}$ ketotifen ${ }^{[13]}$ ) can be selected for treatment. In recent years, it has been reported that leukotriene receptor antagonist is also a new treatment option. ${ }^{[14]}$

\section{Conclusion}

It is highly argued that performing gastrointestinal endoscopy and biopsy can be conducive to the diagnosis of EG in the patients with liver cirrhosis associated with peripheral blood or ascetic fluid eosinophilia. Multiple pathological samples should be taken to definite diagnosis for fear of missing the lesions. We should consider the possibility of EP and perform laparoscopy or peritoneal puncture biopsy when 
nothing special is found in gastrointestinal endoscopy biopsy. other geographic areas and ethnic groups.

Given the limited number of cases reported in the literature, further studies are needed to generalize these results among

\section{CONFlicts OF INTEREST Disclosure}

The authors declare that they have no competing interest.

\section{REFERENCES}

[1] Rothenberg ME. Eosinophilic gastrointestinal disorders (EGID). J Allergy Clin Immunol. 2004; 113(1): 11-28; quiz 29. PMid:14713902. https://doi.org/10.1016/j.jaci.2003.10.047

[2] Pineton de Chambrun G, Gonzalez F, Canva JY, et al. Natural history of eosinophilic gastroenteritis. Clinical gastroenterology and hepatology: the official clinical practice journal of the American Gastroenterological Association. 2011; 9(11): 950-56.e1. PMid:21806952. https://doi.org/10.1016/j.cgh.2011.07.017

[3] Klein NC, Hargrove RL, Sleisenger MH, et al. Eosinophilic gastroenteritis. Medicine (Baltimore). 1970; 49(4): 299-319. https: //doi.org/10.1097/00005792-197007000-00003

[4] Ingle SB, Hinge Ingle CR. Eosinophilic gastroenteritis: an unusual type of gastroenteritis. World J Gastroenterol. 2013; 19(31): 5061-6. PMid:23964139. https://doi.org/10.3748/wjg.v19.i31.50 61

[5] Santarpia L, Cuomo R, Camera L, et al. A patient with oeosinophilic gastroenteritis and severe malnutrition improved with home parenteral nutrition. BMJ Case Reports. 2010; 2010: PMid:22798439. https://doi.org/10.1136/bcr.04.2010.2928

[6] Oh HE, Chetty R. Eosinophilic gastroenteritis: a review. Journal of Gastroenterology. 2008; 43(10): 741-50. PMid:18958542. https://doi.org/10.1007/s00535-008-2230-5

[7] Talley NJ, Shorter RG, Phillips SF, et al. Eosinophilic gastroenteritis: a clinicopathological study of patients with disease of the mucosa, muscle layer, and subserosal tissues. Gut. 1990; 31(1): 54-8. PMid:2318432. https://doi.org/10.1136/gut.31.1.54

[8] Xu Y, Gao C, Xu J, et al. Successful Treatment of Idiopathic Eosinophilic Peritonitis by Oral Corticosteroid Therapy in a Continu- ous Ambulatory Peritoneal Dialysis Patient. Case Rep Nephrol Dial. 2015; 5(2): 130-4. PMid:26266243. https://doi .org/10.1159/ 000431085

[9] Ishihara S, Kinoshita Y, Schoepfer A. Eosinophilic Esophagitis, Eosinophilic Gastroenteritis, and Eosinophilic Colitis: Common Mechanisms and Differences between East and West. Inflammatory intestinal diseases. 2016; 1(2): 63-69. PMid:29922659. https : //doi.org/10.1159/000445131

[10] Maleki N, Kalantar Hormozi M, Bahtouee M, et al. Eosinophilic ascites and duodenal obstruction in a patient with liver cirrhosis. Case Rep Gastrointest Med. 2014; 2014: 928496. PMid:24772356. https://doi.org/10.1155/2014/928496

[11] Campos Ruiz A, Ganchegui Aguirre I, Urtasun Arlegui L, et al. Eosinophilic gastroenteritis: three clinical cases with different presentations. Gastroenterologia y Hepatologia. 2017; 40(4): 327-29. https://doi.org/10.1016/j.gastre.2017.03.009

[12] Thakur SS, Unikowsky B, Prichard S. Eosinophilic peritonitis in CAPD: treatment with prednisone and diphenhydramine. Peritoneal dialysis international: journal of the International Society for Peritoneal Dialysis. 1997; 17(4): 402-3.

[13] Tang S, Lo CY, Lo WK, et al. Resolution of eosinophilic peritonitis with ketotifen. American journal of kidney diseases: the official Journal of the National Kidney Foundation. 1997; 30(3): 433-6. https ://doi.org/10.1016/S0272-6386 (97) 90290-0

[14] Forbes TA, Lunn AJ. Montelukast: a novel therapeutic option in eosinophilic peritonitis. Pediatric nephrology (Berlin, Germany) 2014; 29(7): 1279-82. PMid:24346824. https://doi .org/10.1 $007 / \mathrm{s} 00467-013-2718-\mathrm{y}$ 